\title{
On the solutions of second order generalized difference equations
}

\author{
M Maria Susai Manuel' ${ }^{1}$, Adem Kılıçman²*, G Britto Antony Xavier ${ }^{3}$, R Pugalarasu $^{3}$ and DS Dilip ${ }^{3}$
}

"Correspondence:

akilicman@putra.upm.edu.my

${ }^{2}$ Department of Mathematics and Institute for Mathematical Research,

University Putra Malaysia, Serdang, Selangor 43400, Malaysia

Full list of author information is available at the end of the article

\begin{abstract}
In this article, the authors discuss $\ell_{2(\ell)}$ and $c_{0(\ell)}$ solutions of the second order generalized difference equation

$$
\Delta_{\ell}^{2} u(k)+f(k, u(k))=0, \quad k \in[a, \infty), a>0
$$

and we prove the condition for non existence of non-trivial solution where $\Delta_{\ell} u(k)=u(k+\ell)-u(k)$ for $\ell>0$. Further we present some formulae and examples to find the values of finite and infinite series in number theory as application of $\Delta_{\ell}$.

MSC: 39A12; 39A70; 47B39; 39B60
\end{abstract}

Keywords: generalized difference equation; generalized difference operator

\section{Introduction}

Difference equations usually describe the evolution of some certain phenomena over time and are also important in describing dynamics for fundamentally discrete system, see [1]. For example, in the numerical integration, the standard approach is to use the difference equations. Similarly, the population dynamics have discrete generations; the size of the $(k+1)$ st generation $u(k+1)$ is a function of the $k$ th generation $u(k)$. This can be expressed as difference equation of the form

$$
u(k+1)=f(u(k))
$$

see for example [2]. Further, the concept of difference equations with many examples in applications such as asymptotic behavior of solutions of difference equations were studied extensively by Elaydi [3] where the analytic and geometric approaches were also combined in order to studying difference equations. Further, in [3], both classical and modern treatment of the difference equations were presented in excellent form. For related results on difference equations, see [4-8]. In the present article, we study $\ell_{2(\ell)}$ and $c_{0(\ell)}$ solutions of the following second order generalized difference equation

$$
\Delta_{\ell}^{2} u(k)+f(k, u(k))=0, \quad k \in[a, \infty), a>0,
$$

where $\Delta_{\ell} u(k)=u(k+\ell)-u(k)$ for $\ell>0$. We provide some related definitions and development for the present article.

( 2012 Manuel et al.; licensee Springer. This is an Open Access article distributed under the terms of the Creative Commons Attribution License (http://creativecommons.org/licenses/by/2.0), which permits unrestricted use, distribution, and reproduction in any medium, provided the original work is properly cited. 
The basic theory of difference equations is based on the operator $\Delta$ defined as

$$
\Delta u(k)=u(k+1)-u(k), \quad k \in \mathbb{N},
$$

where $\mathbb{N}=\{0,1,2,3, \ldots$,$\} . Even though many authors [1-4] have suggested the definition$ of $\Delta$ as

$$
\Delta u(k)=u(k+\ell)-u(k), \quad k \in \mathbb{N}, \ell \in \mathbb{R}-\{0\}
$$

and there are several research took place on this line. By defining $\Delta_{\ell}$ and its inverse $\Delta_{\ell}^{-1}$, many interesting results and applications in number theory as well as in fluid dynamics can be obtained. By extending the study for sequences of complex numbers and $\ell$ to be real, some new qualitative properties like rotatory, expanding, shrinking, spiral and weblike structures were studied for the solutions of difference equations involving $\Delta_{\ell}$. For similar results, we refer to [9-13].

In particular, the $\ell_{2}$ and $c_{0}$ solutions of second order difference equations of (1) when $\ell=1$, were discussed in [8]. In this article, we discuss $\ell_{2(\ell)}$ and $c_{0(\ell)}$ solutions for the second order generalized difference Equation (1) and present some applications of $\Delta_{\ell}$ in the finite and infinite series of number theory. Throughout this article, we use the following notation:

(i) $[k]$ denotes the integer part of $k$,

(ii) $\mathbb{N}=\{0,1,2,3, \ldots\}, \mathbb{N}(a)=\{a, a+1, a+2, \ldots\}$,

(iii) $\mathbb{N}_{\ell}(j)=\{j, j+\ell, j+2 \ell, \ldots\}$ and $\mathbb{R}$ is the set of all real numbers.

\section{Preliminaries}

In this section, we present some of the preliminary definitions and related results which will be useful for future discussion. The following three definitions held in [9].

Definition 2.1 Let $u:[0, \infty) \rightarrow \mathbb{C}$ and $\ell \in(0, \infty)$ then, the generalized difference operator $\Delta_{\ell}$ is defined as

$$
\Delta_{\ell} u(k)=u(k+\ell)-u(k) .
$$

Similarly, the generalized difference operator of the $r$ th kind is defined as

$$
\Delta_{\ell}^{r}=\Delta_{\ell}\left(\Delta_{\ell}^{r-1}\right) \quad \text { if } r \geq 2 .
$$

Definition 2.2 For arbitrary $x, y \in \mathbb{R}$ the $h$-factorial function is defined by

$$
x_{h}^{(y)}=h^{y} \frac{\Gamma\left(\frac{x}{h}+1\right)}{\Gamma\left(\frac{x}{h}+1-y\right)}
$$

where $\Gamma$ is the Euler gamma function. Note that when $x=k, h=\ell, y=n \in \mathbb{N}(1)$ Definition 2.2 coincides with Definition 2.1.

Definition 2.3 Let $u(k), k \in[0, \infty)$ be a real or complex valued function and $\ell \in(0, \infty)$. Then, the inverse of $\Delta_{\ell}$ denoted by $\Delta_{\ell}^{-1}$ and defined as follows.

$$
\text { If } \Delta_{\ell} v(k)=u(k) \text {, then } v(k)=\Delta_{\ell}^{-1} u(k)+c_{j} \text {, }
$$


where $c_{j}$ is a constant for all $k \in \mathbb{N}_{\ell}(j), j=k-\left\lceil\frac{k}{\ell}\right\rceil \ell$.

Definition 2.4 The generalized polynomial factorial for $\ell>0$ is defined as

$$
k_{\ell}^{(n)}=k(k-\ell)(k-2 \ell) \cdots(k-(n-1) \ell) .
$$

Lemma 2.5 If $\ell>0$ and $n \in \mathbb{N}_{\ell}(1)$ then,

$$
\Delta_{\ell}^{-1} k_{\ell}^{(n)}=\frac{1}{(n+1) \ell}(k-\ell)_{\ell}^{(n+1)}+c_{j}
$$

for all $k \in \mathbb{N}_{\ell}(j), j=k-\left\lceil\frac{k}{\ell}\right\rceil \ell$ and $c_{j}$ is constant.

Lemma 2.6 ([13] Product formula) Let $u(k)$ and $v(k)$ be any two functions. Then

$$
\begin{aligned}
\Delta_{\ell}\{u(k) v(k)\} & =u(k+\ell) \Delta_{\ell} v(k)+v(k) \Delta_{\ell} u(k) \\
& =v(k+\ell) \Delta_{\ell} u(k)+u(k) \Delta_{\ell} v(k), \quad \forall k \in \mathbb{N}_{\ell}(a) .
\end{aligned}
$$

Lemma 2.7 ([12]) Let $\ell>0, n \in \mathbb{N}(2), k \in(\ell, \infty)$ and $k_{\ell}^{(n)} \neq 0$. Then,

$$
\Delta_{\ell}^{-1} \frac{1}{k_{\ell}^{(n)}}=\frac{-1}{(n-1) \ell(k-\ell)_{\ell}^{(n-1)}}+c_{j}
$$

Definition 2.8 A function $u(k), k \in[a, \infty)$ is said to be in the space $\ell_{2(\ell)}$, if

$$
\sum_{\gamma=0}^{\infty}|u(a+j+\gamma \ell)|^{2}<\infty \quad \text { for all } j \in[0, \ell)
$$

If $\lim _{r \rightarrow \infty}|u(a+j+r \ell)|=0$, for all $0 \leq j<\ell$ then $u(k)$ is said to be in the space $c_{0(\ell)}$.

Lemma 2.9 ([9] Summation formula of finite series) If real valued function $u(k)$ is defined for all $k \in[0, \infty)$, then

$$
\Delta_{\ell}^{-1} u(k)=\sum_{r=1}^{\left\lceil\frac{k}{\ell}\right\rceil} u(k-r \ell)+c_{j},
$$

where $c_{j}$ is a constantfor all $k \in \mathbb{N}_{\ell}(j), j=k-\left\lceil\frac{k}{\ell}\right\rceil \ell$. Since $[0, \infty)=\bigcup_{0 \leq j<\ell} N_{\ell}(j)$, each complex number $c_{j},(0 \leq j<\ell)$ is called an initial value of $k \in N_{\ell}(j)$. Usually, each initial value $c_{j}$ is taken from any one of the values $u(j), u(j+\ell), u(j+2 \ell)$, etc.

Lemma 2.10 (Summation formula of infinite series) If $\lim _{k \rightarrow \infty} u(k)=0$ and $\ell>0$, then

$$
\Delta_{\ell}^{-1} u(k)=-\sum_{r=0}^{\infty} u(k+r \ell)
$$


Proof Assume $z(k)=\sum_{r=0}^{\infty} u(k+r \ell)$. Then,

$$
\Delta_{\ell} z(k)=\sum_{r=0}^{\infty} u(k+\ell+r \ell)-\sum_{r=0}^{\infty} u(k+r \ell)=-u(k) .
$$

Now, the proof follows from $\lim _{k \rightarrow \infty} u(k)=0$ and Definition 2.3.

Theorem 2.11 If $\lim _{k \rightarrow \infty} u(k)=0$ and $\ell>0$, then

$$
\Delta_{\ell}^{-2} u(k)=\sum_{r_{1}=0}^{\infty} \sum_{r_{2}=0}^{\infty} u\left(k+r_{1} \ell+r_{2} \ell\right)
$$

Proof The proof follows by taking $\Delta_{\ell}^{-1}$ on (14).

Corollary 2.12 Let $k \in[\ell, \infty)$ and $\ell \in(0, \infty)$. Then

$$
\Delta_{\ell}^{-1} \frac{1}{k(k-\ell)}=-\frac{1}{\ell(k-\ell)}
$$

and hence

$$
\sum_{r=0}^{\infty} \frac{1}{(k+r \ell)(k+r \ell-\ell)}=\frac{1}{\ell(k-\ell)}
$$

Proof The proof follows from Equation (14) and $c_{j}=0$ as $k \rightarrow \infty$.

The following example illustrates Corollary 2.12

Example 2.13 Taking $\ell=0.8, k=1$ in (16), we obtain

$$
\frac{1}{1 \times 0.2}+\frac{1}{1.8 \times 1}+\frac{1}{2.6 \times 1.8}+\cdots=\frac{1}{0.8 \times 0.2}
$$

The following example shows that $\frac{1}{k_{\ell}^{(n)}} \in c_{0(\ell)}$ and $\ell_{2(\ell)}$.

Example 2.14 Assume $n \in \mathbb{N}(2)$ and $k \in[n \ell, \infty)$. Let $u(k)=\frac{1}{k_{\ell}^{(n)}}$. By Lemmas 2.7 and 2.10, we obtain

$$
\frac{1}{(n-1) \ell k_{\ell}^{(n-1)}}=\sum_{r=0}^{\infty} \frac{1}{(k+r \ell)_{\ell}^{(n)}} .
$$

Since $c_{j}=0$ as $k \rightarrow \infty$. Replacing $k$ by $a+j$, we get

$$
\sum_{r=0}^{\infty} \frac{1}{(a+j+r \ell)_{\ell}^{(n)}}=\frac{1}{(n-1) \ell(a+j)_{\ell}^{(n-1)}}, \quad \text { for } a \geq n \ell .
$$

Since

$$
\left|\frac{1}{(a+j+r \ell)_{\ell}^{(n)}}\right|^{2}<\frac{1}{(a+j+r \ell)_{\ell}^{(n)}},
$$


for $a \geq n \ell$ thus Equation (17) yields

$$
\sum_{r=0}^{\infty}|u(a+j+r \ell)|^{2}<\sum_{r=0}^{\infty} \frac{1}{(a+j+r \ell)_{\ell}^{(n)}}=\frac{1}{(n-1) \ell(a+j)^{(n-1)}}<\infty .
$$

By Definition 2.8, the function $\frac{1}{k_{\ell}^{(n)}} \in \ell_{2(\ell)}$. Since

$$
\lim _{r \rightarrow \infty} \frac{1}{(a+j+r \ell)_{\ell}^{(n)}}=0, \quad \frac{1}{k_{\ell}^{(n)}} \in c_{0(\ell)} .
$$

Now taking $a=n \ell$ then $u(k)$ is an $\ell_{2(\ell)}$ space function.

\section{Main results}

In this section, we present the condition for non existence of non-trivial solution of (1).

Lemma 3.1 Let $a \geq 2 \ell$ and $k \in[a, \infty)$. Then

$$
\frac{1}{k}<\frac{4}{(\sqrt{k+\ell}+\sqrt{k})(\sqrt{k}+\sqrt{k-\ell})} .
$$

Proof We have

$$
\begin{aligned}
\frac{4}{(\sqrt{k+\ell}+\sqrt{k})(\sqrt{k}+\sqrt{k-\ell})} \\
=\frac{4(\sqrt{k+\ell}-\sqrt{k})(\sqrt{k}-\sqrt{k-\ell})}{\ell^{2}} \\
=\frac{4}{\ell^{2}} \sqrt{k} \sqrt{k}\left[\left(1+\frac{\ell}{k}\right)^{\frac{1}{2}}-1\right]\left[1-\left(1-\frac{\ell}{k}\right)^{\frac{1}{2}}\right] \\
=\frac{4 k}{\ell^{2}}\left[1+\frac{1}{2} \frac{\ell}{k}-\frac{1}{2 !} \frac{1}{4}\left(\frac{\ell}{k}\right)^{2}+\frac{1}{3 !} \frac{1}{4} \frac{3}{2}\left(\frac{\ell}{k}\right)^{3}-\frac{1}{4 !} \frac{1}{4} \frac{3}{2} \frac{5}{2}\left(\frac{\ell}{k}\right)^{4}+\cdots\right] \\
\quad \times\left[1-\left(1-\frac{1}{2} \frac{\ell}{k}-\frac{1}{2 !} \frac{1}{4}\left(\frac{\ell}{k}\right)^{2}-\frac{1}{3 !} \frac{1}{4} \frac{3}{2}\left(\frac{\ell}{k}\right)^{3}-\frac{1}{4 !} \frac{1}{4} \frac{3}{2} \frac{5}{2}\left(\frac{\ell}{k}\right)^{4}-\cdots\right)\right] .
\end{aligned}
$$

Since each positive term is greater than the consecutive negative term in the first expression, we find

$$
\begin{aligned}
& \frac{4 k}{\ell^{2}}\left[\frac{1}{2} \frac{\ell}{k}-\frac{1}{2 !} \frac{1}{4}\left(\frac{\ell}{k}\right)^{2}\right] \times\left[\frac{1}{2} \frac{\ell}{k}+\frac{1}{2 !} \frac{1}{4}\left(\frac{\ell}{k}\right)^{2}+\frac{1}{3 !} \frac{1}{4} \frac{3}{2}\left(\frac{\ell}{k}\right)^{3}+\frac{1}{4 !} \frac{1}{4} \frac{3}{2} \frac{5}{2}\left(\frac{\ell}{k}\right)^{4}+\cdots\right] \\
&=\frac{4}{\ell^{2}}\left[\frac{\ell}{2}-\frac{\ell}{2} \frac{1}{4} \frac{\ell}{k}\right]\left[\frac{1}{2} \frac{\ell}{k}+\frac{1}{2 !} \frac{1}{4}\left(\frac{\ell}{k}\right)^{2}+\frac{1}{3 !} \frac{1}{4} \frac{3}{2}\left(\frac{\ell}{k}\right)^{3}+\frac{1}{4 !} \frac{1}{4} \frac{3}{2} \frac{5}{2}\left(\frac{\ell}{k}\right)^{4}+\cdots\right] \\
&=\frac{4}{\ell^{2}} \frac{\ell}{2}\left[\frac{1}{2} \frac{\ell}{k}+\frac{1}{2 !} \frac{1}{4}\left(\frac{\ell}{k}\right)^{2}+\frac{1}{3 !} \frac{1}{4} \frac{3}{2}\left(\frac{\ell}{k}\right)^{3}+\frac{1}{4 !} \frac{1}{4} \frac{3}{2} \frac{5}{2}\left(\frac{\ell}{k}\right)^{4}+\cdots\right] \\
&-\frac{4}{\ell^{2}} \frac{\ell}{2} \frac{1}{4} \frac{\ell}{k}\left[\frac{1}{2} \frac{\ell}{k}+\frac{1}{2 !} \frac{1}{4}\left(\frac{\ell}{k}\right)^{2}+\frac{1}{3 !} \frac{1}{4} \frac{3}{2}\left(\frac{\ell}{k}\right)^{3}+\frac{1}{4 !} \frac{1}{4} \frac{3}{2} \frac{5}{2}\left(\frac{\ell}{k}\right)^{4}+\cdots\right] \\
&= \frac{1}{k}+\frac{2}{\ell}\left[\frac{1}{2 !} \frac{1}{4}\left(\frac{\ell}{k}\right)^{2}+\frac{1}{3 !} \frac{1}{4} \frac{3}{2}\left(\frac{\ell}{k}\right)^{3}+\frac{1}{4 !} \frac{1}{4} \frac{3}{2} \frac{5}{2}\left(\frac{\ell}{k}\right)^{4}+\cdots\right]
\end{aligned}
$$




$$
\begin{aligned}
& -\frac{2}{\ell}\left[\frac{1}{2 !} \frac{1}{4}\left(\frac{\ell}{k}\right)^{2}+\frac{1}{2 !} \frac{1}{4} \frac{1}{4}\left(\frac{\ell}{k}\right)^{3}+\frac{1}{3 !} \frac{1}{4} \frac{1}{4} \frac{1}{4}\left(\frac{\ell}{k}\right)^{4}+\cdots\right] \\
= & \frac{1}{k}+\frac{2}{4 \ell}\left[\frac{1}{3 !}\left(\frac{3}{2}-\frac{3}{4}\right)\left(\frac{\ell}{k}\right)^{3}+\frac{1}{4 !} \frac{3}{2}\left(\frac{5}{2}-\frac{4}{4}\right)\left(\frac{\ell}{k}\right)^{4}\right. \\
& \left.+\frac{1}{5 !} \frac{3}{2} \frac{5}{2}\left(\frac{7}{2}-\frac{5}{4}\right)\left(\frac{\ell}{k}\right)^{5}+\frac{1}{6 !} \frac{3}{2} \frac{5}{2} \frac{7}{2}\left(\frac{9}{2}-\frac{6}{4}\right)\left(\frac{\ell}{k}\right)^{6}+\cdots\right]>\frac{1}{k},
\end{aligned}
$$

since the second term is positive.

Lemma 3.2 Let $a \geq 2 \ell$ and $k \in[a, \infty)$. Then

$$
\frac{\sqrt{k+\ell}}{\sqrt{k}}-\frac{\sqrt{k}}{\sqrt{k+\ell}+\sqrt{k-\ell}}<1 \text {. }
$$

Proof From the Binomial theorem for rational index, we find

$$
\begin{aligned}
\frac{\sqrt{k+\ell}}{\sqrt{k}}-\frac{\sqrt{k}}{\sqrt{k+\ell}+\sqrt{k-\ell}}= & \left(1+\frac{\ell}{k}\right)^{\frac{1}{2}}-\frac{\sqrt{k}}{2 \ell}\left[(k+\ell)^{\frac{1}{2}}-(k-\ell)^{\frac{1}{2}}\right] \\
= & 1+\frac{1}{2} \frac{\ell}{k}-\frac{1}{2 !} \frac{1}{2} \frac{1}{2}\left(\frac{\ell}{k}\right)^{2}+\frac{1}{3 !} \frac{1}{2} \frac{1}{2} \frac{3}{2}\left(\frac{\ell}{k}\right)^{3}-\cdots \\
& -\frac{k}{2 \ell}\left[1+\frac{1}{2} \frac{\ell}{k}-\frac{1}{2 !} \frac{1}{2} \frac{1}{2}\left(\frac{\ell}{k}\right)^{2}+\frac{1}{3 !} \frac{1}{2} \frac{1}{2} \frac{3}{2}\left(\frac{\ell}{k}\right)^{3}-\cdots\right. \\
& \left.-\left(1-\frac{1}{2} \frac{\ell}{k}-\frac{1}{2 !} \frac{1}{2} \frac{1}{2}\left(\frac{\ell}{k}\right)^{2}-\frac{1}{3 !} \frac{1}{2} \frac{1}{2} \frac{3}{2}\left(\frac{\ell}{k}\right)^{3}-\cdots\right)\right] \\
= & 1+\frac{1}{2} \frac{\ell}{k}-\frac{1}{2 !} \frac{1}{2} \frac{1}{2}\left(\frac{\ell}{k}\right)^{2}+\frac{1}{3 !} \frac{1}{2} \frac{1}{2} \frac{3}{2}\left(\frac{\ell}{k}\right)^{3}-\cdots \\
& -\frac{k}{2 \ell}\left[\frac{\ell}{k}+\frac{1}{3 !} \frac{1}{2} \frac{1}{2} \frac{l}{2}\left(\frac{\ell}{k}\right)^{3}+\cdots\right] .
\end{aligned}
$$

Since each negative terms is greater than the next consecutive positive term and $k \geq 2 \ell$, we get

$$
\frac{\sqrt{k+\ell}}{\sqrt{k}}-\frac{\sqrt{k}}{\sqrt{k+\ell}+\sqrt{k-\ell}}=1+\frac{1}{2} \frac{\ell}{k}-\frac{1}{2}=\frac{1}{2}+\frac{1}{2} \frac{\ell}{k}<1 .
$$

Lemma 3.3 Let $a \geq 2 \ell$. If

$$
\Delta_{\ell} z(k) \leq \alpha(k)+\beta(k) z(k)
$$

and $\frac{-\ell}{k}<\beta<\frac{-\ell^{2}}{k^{2}}$ for all $k \in[a, \infty)$ then

$$
\Delta_{\ell}\left(z(k) \prod_{r=0}^{\left\lceil\frac{k-a}{\ell}\right\rceil-1}(1+\beta(j+a+r \ell))^{-1}\right) \leq \alpha(k) \prod_{r=0}^{\left\lceil\frac{k-a}{\ell}\right\rceil}(1+\beta(j+a+r \ell))^{-1},
$$

where $j=k-a-\left\lceil\frac{k-a}{\ell}\right\rceil \ell$. 
Proof From the inequality (19) and $1+\beta(k)>0$ for all $k \in[a, \ell)$, we find,

$$
\frac{z(k+\ell)}{1+\beta(k)}-z(k) \leq \frac{\alpha(k)}{1+\beta(k)}
$$

which yields,

$$
\begin{aligned}
& \frac{z(k+\ell)}{1+\beta(k)} \prod_{r=0}^{\left\lceil\frac{k-a}{\ell}\right\rceil-1}(1+\beta(j+a+r \ell))^{-1}-z(k) \prod_{r=0}^{\left\lceil\frac{k-a}{\ell}\right\rceil-1}(1+\beta(j+a+r \ell))^{-1} \\
& \leq \frac{\alpha(k)}{1+\beta(k)} \prod_{r=0}^{\left\lceil\frac{k-a}{\ell}\right\rceil-1}(1+\beta(j+a+r \ell))^{-1} .
\end{aligned}
$$

Now (20) follows by taking $r=\left\lceil\frac{k-a}{\ell}\right\rceil$ and $j+a+\left\lceil\frac{k-a}{\ell}\right\rceil \ell=k$.

The following theorem shows the nonexistence of solutions of (3).

Theorem 3.4 For all $(k, u) \in[a, \infty) \times \mathbb{R}$, let the function $f(k, u)$ be defined and

$$
|f(k, u)| \leq \frac{\ell^{2}}{2} k^{-2}|u|
$$

Then, if $u(k) \in \ell_{2(\ell)}$ is a solution of (3), there exists a real $k_{1} \geq a(a \geq 2 \ell)$ such that $u(k)=0$ for all $k \in\left[k_{1}, \infty\right)$.

Proof Since $u(k)$ is a solution of (3) and belong to $\ell_{2(\ell)}$, we have $\sum_{r=0}^{\infty}|u(a+j+r \ell)|^{2}<\infty$ which yields $\lim _{k \rightarrow \infty} u(k)=0$ and hence

$$
\lim _{k \rightarrow \infty} \Delta_{\ell} u(k)=\lim _{k \rightarrow \infty} \Delta_{\ell}^{2} u(k)=0
$$

By using Equations (3) and (22), and applying $\Delta_{\ell}^{-1}$ on Equation (3) with Lemma 2.10, we obtain

$$
\Delta_{\ell} u(k)=\sum_{r=0}^{\infty} f(k+r \ell, u(k+r \ell)) .
$$

Now by applying again $\Delta_{\ell}^{-1}$ on both sides, and by Theorem 2.10 , we get

$$
u(k)=-\sum_{r=0}^{\infty} \sum_{s=0}^{\infty} f(k+r \ell+s \ell, u(k+r \ell+s \ell))
$$

which yields

$$
u(k)=-\sum_{r=0}^{\infty}(r+1) f(k+r \ell, u(k+r \ell)), \quad k \in[a, \infty)
$$

Therefore, from (21), we obtain

$$
|u(k)| \leq \frac{\ell^{2}}{2} v(k)
$$


where

$$
v(k)=\sum_{r=0}^{\infty}(r+1)(k+r \ell)^{-2}|u(k+r \ell)|, \quad \text { for all } k \in[a, \infty) .
$$

Obviously $v(k) \geq 0$ for all $k \in[a, \infty)$ and $\lim _{k \rightarrow \infty} v(k)=0$.

If $v(k+j)=0, \forall j \in[0, \ell)$, for some $k=k_{1} \geq a$, then $(r+1)(k+j+r \ell)^{-2} u(k+j+r \ell)=0$ for all $r=0,1,2, \ldots$. Hence $u(k)=0$ for all $k \geq k_{1}$. In this case, the proof is complete.

Now, we suppose that $v(k)>0$ for all $k \in[a, \infty)$, from (27), we have

$$
\Delta_{\ell} v(k)=-\sum_{r=0}^{\infty}(k+r \ell)^{-2}|u(k+r \ell)|
$$

and

$$
\Delta_{\ell}^{2} v(k)=k^{-2}|u(k)| .
$$

From (26), we have

$$
\Delta_{\ell}^{2} v(k) \leq \frac{\ell^{2}}{2} k^{-2} v(k) \quad \text { for all } k \in[a, \infty) .
$$

From (27), $a \geq 2 \ell, \frac{r+1}{k+r \ell} \leq \frac{1}{\ell}$, by Schwartz's inequality, we obtain

$$
v(k) \leq \ell^{-1} \sum_{r=0}^{\infty}(k+r \ell)^{-1}|u(k+r \ell)| \leq \ell^{-1}\left(\sum_{r=0}^{\infty}(k+r \ell)^{-2}\right)^{\frac{1}{2}}\left(\sum_{r=0}^{\infty}|u(k+r \ell)|^{2}\right)^{\frac{1}{2}} .
$$

By using Corollary 2.12, we get

$$
v(k) \leq \ell^{-\frac{3}{2}} \frac{1}{\sqrt{k-\ell}}\left(\sum_{r=0}^{\infty}|u(k+r \ell)|^{2}\right)^{\frac{1}{2}} .
$$

If $w(k)=\ell^{\frac{3}{2}} \sqrt{k-\ell} v(k)$, then

$$
w(k) \leq\left(\sum_{r=0}^{\infty}\|u(k+r \ell)\|^{2}\right)^{\frac{1}{2}}, \quad \text { for all } k \in[a, \infty) .
$$

Hence we have

$$
w(k) \rightarrow 0 \quad \text { and } \quad w(k)>0, \quad \forall k \in[a, \infty) .
$$

By applying Lemma 2.6 to Equation (29) twice, we obtain

$$
\Delta_{\ell}^{2} w(k)=\ell^{\frac{3}{2}}\left(\sqrt{k+\ell} \Delta_{\ell}^{2} v(k)+2 \Delta_{\ell} v(k) \Delta_{\ell} \sqrt{k}+v(k) \Delta_{\ell}^{2} \sqrt{k-\ell}\right) .
$$

Again from Lemma 2.6 and Equation (29), we obtain

$$
\Delta_{\ell} v(k)=\ell^{-\frac{3}{2}}\left(\frac{1}{\sqrt{k}} \Delta_{\ell} w(k)+w(k) \Delta_{\ell} \frac{1}{\sqrt{k-\ell}}\right) .
$$


From (31), (32) and by Lemma 2.6, we find that

$$
\begin{aligned}
& \Delta_{\ell}\left(\frac{1}{k-\ell} \Delta_{\ell} w(k)\right) \\
& =\frac{1}{k} \Delta_{\ell}^{2} w(k)-\left(\frac{\ell}{k(k-\ell)}\right) \Delta_{\ell} w(k) \\
& =\frac{\ell^{\frac{3}{2}}}{k}\left\{\sqrt{k+\ell} \Delta_{\ell}^{2} v(k)+2 \Delta_{\ell} v(k) \Delta_{\ell} \sqrt{k}+v(k) \Delta_{\ell}^{2} \sqrt{k-\ell}\right\} \\
& -\left(\frac{\ell}{k(k-\ell)}\right) \Delta_{\ell} w(k) \\
& =\frac{\ell^{\frac{3}{2}}}{k}\left\{\sqrt{k+\ell} \Delta_{\ell}^{2} v(k)+2 \ell^{-\frac{3}{2}}\left[\frac{1}{\sqrt{k}} \Delta_{\ell} w(k)+2 \frac{w(k)}{k} \Delta_{\ell} \frac{1}{\sqrt{k-\ell}}\right] \Delta_{\ell} \sqrt{k}\right. \\
& \left.+\frac{\ell^{\frac{3}{2}}}{k} v(k) \Delta_{\ell}^{2} \sqrt{k-\ell}\right\}-\left(\frac{\ell}{k(k-\ell)}\right) \Delta_{\ell} w(k) \\
& =\ell^{\frac{3}{2}}\left(\frac{\sqrt{k+\ell}}{k}\right) \Delta_{\ell}^{2} \nu(k)+\frac{2}{k} \ell^{\frac{3}{2}} \sqrt{k-\ell} \nu(k) \Delta_{\ell} \frac{1}{\sqrt{k-\ell}} \Delta_{\ell} \sqrt{k} \\
& +\frac{\ell^{\frac{3}{2}}}{k} v(k) \Delta_{\ell}^{2} \sqrt{k-\ell}+\frac{2}{k \sqrt{k}} \Delta_{\ell} w(k) \Delta_{\ell} \sqrt{k}-\frac{\ell}{k(k-\ell)} \Delta_{\ell} w(k) \\
& \leq \ell^{\frac{3}{2}}\left(\frac{\ell^{2} \sqrt{k+\ell}}{2 k^{3}}\right) v(k)+\frac{2 \ell^{\frac{3}{2}}}{k} \sqrt{k-\ell} v(k) \Delta_{\ell} \sqrt{k} \Delta_{\ell} \frac{1}{\sqrt{k-\ell}} \\
& +\frac{\ell^{\frac{3}{2}}}{k} v(k) \Delta_{\ell}^{2} \sqrt{k-\ell} \\
& +\left(\frac{2(k-\ell)}{k \sqrt{k}} \Delta_{\ell} \sqrt{k}-\frac{\ell}{k}\right) \frac{1}{k-\ell} \Delta_{\ell} w(k)
\end{aligned}
$$

which in view of (28), (30) gives

$$
\Delta_{\ell} z(k) \leq \alpha(k)+\beta(k) z(k)
$$

where

$$
\begin{aligned}
& z(k)=\frac{1}{k-\ell} \Delta_{\ell} w(k), \\
& \alpha(k)=\ell^{\frac{3}{2}}\left(\frac{\ell^{2} \sqrt{k+\ell}}{2 k^{3}}+\frac{2}{k} \sqrt{k-\ell} \Delta_{\ell} \sqrt{k} \Delta_{\ell} \frac{1}{\sqrt{k-\ell}}+\frac{1}{k} \Delta_{\ell}^{2} \sqrt{k-\ell}\right) \nu(k)
\end{aligned}
$$

and

$$
\beta(k)=\left(\frac{2(k-\ell)}{k \sqrt{k}}\right) \Delta_{\ell} \sqrt{k}-\frac{\ell}{k} .
$$

Since $\left(\frac{2(k-\ell)}{k \sqrt{k}}\right) \Delta_{\ell} \sqrt{k}>0$, from $\left(1+\frac{\ell}{k}\right)^{\frac{1}{2}}<1+\frac{1}{2} \frac{\ell}{k}$, we obtain

$$
-\frac{\ell}{k}<\beta(k)<-\frac{\ell^{2}}{k^{2}}, \quad \text { where } k \in[a, \infty) .
$$


Further, since

$$
\begin{aligned}
\sqrt{k} \sqrt{k-\ell} \Delta_{\ell} \sqrt{k} \Delta_{\ell} \frac{1}{\sqrt{k-\ell}} & =(\sqrt{k+\ell}-\sqrt{k})(\sqrt{k-\ell}-\sqrt{k}) \\
& =-\frac{\ell^{2}}{(\sqrt{k+\ell}+\sqrt{k})(\sqrt{k-\ell}+\sqrt{k})}
\end{aligned}
$$

and

$$
\begin{aligned}
& \Delta_{\ell}^{2} \sqrt{k-\ell}=\sqrt{k+\ell}-\sqrt{k}+\sqrt{k-\ell}-\sqrt{k} \\
& =\frac{(\sqrt{k+\ell}-\sqrt{k})(\sqrt{k+\ell}+\sqrt{k})}{(\sqrt{k+\ell}+\sqrt{k})}+\frac{(\sqrt{k-\ell}-\sqrt{k})(\sqrt{k-\ell}+\sqrt{k})}{(\sqrt{k-\ell}+\sqrt{k})} \\
& =\ell \frac{\sqrt{k-\ell}-\sqrt{k+\ell}}{(\sqrt{k+\ell}+\sqrt{k})(\sqrt{k-\ell}+\sqrt{k})} \\
& \gamma(k)=\frac{\ell^{\frac{3}{2}}}{k \sqrt{k}}\left(\frac{\ell^{2} \sqrt{k+\ell}}{2 k \sqrt{k}}+\frac{-2 \ell^{2}+\ell \sqrt{k}(\sqrt{k-\ell}-\sqrt{k+\ell})}{(\sqrt{k+\ell}+\sqrt{k})(\sqrt{k}+\sqrt{k-\ell})}\right) v(k) .
\end{aligned}
$$

From Lemmas 3.1 and 3.2

$$
\begin{aligned}
\gamma(k)< & \frac{\ell^{\frac{3}{2}}}{k \sqrt{k}}\left(\frac{\ell^{2} \sqrt{k+\ell}}{2 \sqrt{k}} \frac{4}{(\sqrt{k+\ell}+\sqrt{k})(\sqrt{k}+\sqrt{k-\ell})}\right. \\
& \left.+\frac{-2 \ell^{2}+\ell \sqrt{k}(\sqrt{k-\ell}-\sqrt{k+\ell})}{(\sqrt{k+\ell}+\sqrt{k})(\sqrt{k}+\sqrt{k-\ell})}\right) v(k) \\
= & \frac{2 \ell^{\frac{3}{2}}}{k \sqrt{k}(\sqrt{k+\ell}+\sqrt{k})(\sqrt{k}+\sqrt{k-\ell})}\left(\frac{\ell^{2} \sqrt{k+\ell}}{\sqrt{k}}-\frac{\ell^{2} \sqrt{k}}{\sqrt{k+\ell}+\sqrt{k-\ell}}-\ell^{2}\right) v(k) \\
= & \frac{2 \ell^{\frac{7}{2}}}{k \sqrt{k}(\sqrt{k+\ell}+\sqrt{k})(\sqrt{k}+\sqrt{k-\ell})}\left(\frac{\sqrt{k+\ell}}{\sqrt{k}}-\frac{\sqrt{k}}{\sqrt{k+\ell}+\sqrt{k-\ell}}-1\right) v(k) .
\end{aligned}
$$

By Lemma 3.2, we find $\gamma(k)<0$ for all $k \in[a, \infty)$. Thus from Lemma 3.3 and $\gamma(k)<0$, we find

$$
\Delta_{\ell}\left(z(k) \prod_{r=0}^{\left\lceil\frac{k-a}{\ell}\right\rceil-1}(1+\beta(j+a+r \ell))^{-1}\right)<0, \quad \text { for all } k \in[a+\ell, \infty) .
$$

That is,

$$
z(k) \prod_{r=0}^{\left\lceil\frac{k-a}{\ell}\right\rceil-1}(1+\beta(j+a+r \ell))^{-1}
$$

is decreasing by $\ell$ steps.

If

$$
z(k) \prod_{r=0}^{\left\lceil\frac{k-a}{\ell}\right\rceil-1}(1+\beta(j+a+r \ell))^{-1}>0
$$


for all $k \in[a+\ell, \infty)$, then $z(k)>0$. From (34) we find $\Delta_{\ell} w(k)>0$ and hence $w(k)$ is increasing by $\ell$ steps, but this contradicts (30).

If there exists a real $K \geq a+\ell$ such that

$$
z(K) \prod_{r=0}^{\left\lceil\frac{K-a}{\ell}\right\rceil-1}(1+\beta(j+a+r \ell))^{-1}=p_{j}<0
$$

for all $0 \leq j<\ell$, then

$$
z(k) \prod_{r=0}^{\left\lceil\frac{k-a}{\ell}\right\rceil-1}(1+\beta(j+a+r \ell))^{-1}<p_{j}
$$

for all $k \in[K, \infty)$, that is,

$$
z(k)<p_{j} \prod_{r=0}^{\left\lceil\frac{k-a}{\ell}\right\rceil-1}(1+\beta(j+a+r \ell)) .
$$

However from (37), since $1+\beta(k)>(k-\ell) / k>0$ and $j=k-\left\lceil\frac{k-a}{\ell}\right\rceil \ell$, it follows that $z(k)<$ $p_{j}(j+a-\ell) /(k-\ell)$, and hence from (34), we find $\Delta_{\ell} w(k)<p_{j}(j+a-\ell)$. Further, since

$$
w(k) \rightarrow 0, \quad k \geq K+2 \ell \quad \Rightarrow \quad \frac{1}{\ell}(k-K-\ell) \geq 1
$$

we get $w(k+\ell)<w(k)+p_{j}(j+a-\ell)$ which yields $w(k)<w(k-\ell)+p_{j}(j+a-\ell)$ and hence we get

$$
w(k)<w(K+\ell)+\frac{1}{\ell} p_{j}(j+a-\ell)(k-K-\ell)
$$

for all $k \in[K+2 \ell, \infty)$, since

$$
k \geq K+2 \ell \quad \Rightarrow \quad k-K \geq 2 \ell, \quad \frac{1}{\ell}(k-K-\ell) \geq 1 .
$$

But this implies that $w(k) \rightarrow-\infty$, and again we get a contradiction to (30).

Thus combining the above arguments, we conclude that our assumption $v(k)>0$ for all $k \in[a, \infty)$ is not correct, and this completes the proof.

Theorem 3.5 For all $(k, u) \in[0, \infty) \times \mathbb{R}$, let the function $f(k, u)$ be defined and

$$
|f(k, u)| \leq \ell^{q} k^{-q}|u|, \quad q>\frac{5}{2}
$$

Then, if $u(k)$ is a solution of $(3) \in c_{0(\ell)}$, there exists an integer $k_{1} \geq a(a \geq 4 \ell)$ such that $u(k)=0$ for all $k \in\left[k_{1}, \infty\right)$.

Proof Let $u(k)$ be a solution of (3) such that $\lim _{k \rightarrow \infty}|u(k)|=0$. Then,

$$
\lim _{k \rightarrow \infty} \Delta_{\ell} u(k)=\lim _{k \rightarrow \infty} \Delta_{\ell}^{2} u(k)=0
$$


for all $\ell>0$. Thus, for this solution also the relation (24) holds. Further, since there exists a constant $c_{j}>0$ such that $|u(k)| \leq c_{j}$ for all $k \in[a, \infty)$, where $0 \leq j=k-\left\lceil\frac{k}{\ell}\right\rceil \ell<\ell$, we find that

$$
\begin{aligned}
\sum_{r=0}^{\infty}(r+1)|f((k+r \ell), u(k+r \ell))| & \leq \sum_{r=0}^{\infty}\left(r+\frac{k}{\ell} \ell^{q}(k+r \ell)^{-q}|u(k+r \ell)|\right) \\
& =\sum_{r=0}^{\infty}(k+r \ell)^{1-q} \ell^{q-1}|u(k+r \ell)| \\
& \leq c_{j} \ell^{q-1} \sum_{r=0}^{\infty}(k+r \ell)^{1-q} \quad \text { where } j=k-\left\lceil\frac{k}{\ell}\right] \ell \\
& =c_{j} \ell^{q-1}\left[k^{1-q}+\sum_{r=1}^{\infty}(k+r \ell)^{1-q}\right] \\
& =c_{j} \ell^{q-1}\left[k^{1-q}+\ell^{1-q} \sum_{r=1}^{\infty}\left(\frac{k}{\ell}+r\right)^{1-q}\right] \\
& =c_{j} \ell^{q-1}\left[k^{1-q}+\ell^{1-q}\left[\frac{\left(\frac{k}{\ell}\right)^{2-q}}{2-q}+r\right]_{\frac{k}{\ell}}^{\infty}\right] \\
& =c_{j} \ell^{q-1}\left[k^{1-q}+\left(\frac{k^{2-q}}{\ell(q-2)}\right)\right]<\infty,
\end{aligned}
$$

for all $k \in\left[k_{1}, \infty\right)$. Therefore, this solution also has the representation (24).

Now as in Theorem 3.4, we define

$$
\bar{v}(k)=\sum_{r=0}^{\infty}(r+1)(k+r \ell)^{-q}|u(k+r \ell)|=\sum_{r=0}^{\infty} \ell^{-q}(r+1)\left(\frac{k}{\ell}+r\right)^{-q}|u(k+r \ell)| .
$$

Since $q>\frac{5}{2}$, we find

$$
\bar{v}(k) \leq \ell^{-q} \sum_{r=0}^{\infty}(r+1)\left(\frac{k}{\ell}+r\right)^{-2}|u(k+r \ell)|=\ell^{2-q} \sum_{r=0}^{\infty}(r+1)(k+r)^{-2}|u(k+r \ell)|
$$

then it follows that

$$
\bar{v}(k) \leq \ell^{2-q}\left(\frac{\ell^{-\frac{3}{2}}}{\sqrt{k-\ell}}\right)\left\{\sum_{r=0}^{\infty}|u(k+r \ell)|^{2}\right\}^{\frac{1}{2}} .
$$

Hence we define

$$
\begin{aligned}
& \bar{w}(k)=\ell^{q-\frac{1}{2}} \sqrt{k-\ell} \bar{v}(k), \\
& \bar{z}(k)=\frac{1}{k-\ell} \Delta_{\ell} \bar{w}(k), \\
& \bar{\gamma}(k)=\ell^{q-\frac{1}{2}}\left(\ell^{q} \frac{\sqrt{k+\ell}}{2 k^{q+1}}+\frac{2}{k} \sqrt{k-\ell} \Delta_{\ell} \sqrt{k} \Delta_{\ell} \frac{1}{\sqrt{k-\ell}}+\frac{1}{k} \Delta_{\ell}^{2} \sqrt{k-\ell}\right) \bar{v}(k), \\
& \bar{\beta}(k)=\left(\frac{2(k-\ell)}{k \sqrt{k}}\right) \Delta_{\ell} \sqrt{k}-\frac{\ell}{k},
\end{aligned}
$$


and applying similar arguments as in the previous theorem one can see that there exists a positive integer $k_{1}$ such that $u(k)=0$ for all $k \in\left[k_{1}, \infty\right)$.

In the next we present some formulae and examples to find the values of finite and infinite series in number theory as application of $\Delta_{\ell}$. First of all we need the following theorem.

Theorem 3.6 Let $k \in[\ell, \infty)$ and $\ell \in(0, \infty)$. Then

$$
\sum_{r=1}^{\left[\frac{k}{\ell}\right]+s} \frac{(k-r \ell+2 \ell)^{2}-3 \ell^{2}}{\ell^{r}(k-r \ell+4 \ell)_{\ell}^{(2)}(k-r \ell+\ell)_{\ell}^{\left(\left\lceil\frac{k-r \ell+\ell}{\ell}\right]\right)}}=\frac{c_{j}}{\ell^{\left\lceil\frac{k}{\ell}\right\rceil}}-\frac{1}{(k+3 \ell) k_{\ell}^{\left(\left\lceil\frac{k}{\ell}\right]\right)}},
$$

where $s=-1$ for $k \in \mathbb{N}_{\ell}(\ell), s=0$ for $k \notin \mathbb{N}_{\ell}(\ell)$ and each $c_{j}$ is a constant for all $k \in \mathbb{N}_{\ell}(j)$, $j=k-\left[\frac{k}{\ell}\right] \ell$. In particular $c_{j}$ is obtained from (40) by substituting $k=\ell+j$. Further

$$
\sum_{r=0}^{\infty} \frac{(k+r \ell)^{3}-\ell^{3}}{\ell^{r}\left((k+r \ell)^{2}-2 \ell^{2}\right)_{\ell}^{(2)}(k+r \ell+\ell)_{\ell}^{\left.\left(\frac{\Gamma+r \ell+\ell}{\ell}\right]\right)}}=\frac{1}{\left((k-\ell)^{2}-2 \ell^{2}\right) k_{\ell}^{\left.\left(\Gamma \frac{k}{\ell}\right]\right)}} .
$$

Proof By Definition 2.1, we find

$$
\Delta_{\ell}^{-1} \frac{\left((k+2 \ell)^{2}-3 \ell^{2}\right) \ell^{\left\lceil\frac{k}{\ell}\right\rceil}}{(k+4 \ell)_{\ell}^{(2)}(k+\ell)_{\ell}^{\left(\left\lceil\frac{k+\ell}{\ell}\right\rceil\right)}}=c_{j}-\frac{\ell^{\left\lceil\frac{k}{\ell}\right\rceil}}{(k+3 \ell) k_{\ell}^{\left(\left\lceil\frac{k}{\ell}\right\rceil\right)}}
$$

and (40) follows by Lemma 2.9 and

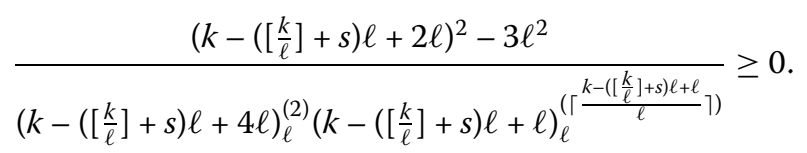

The following example illustrates Theorem 3.6.

Example 3.7 By taking $\ell=1.7, k=2$ and $j=0.3$ in (40), we get $c_{j}=\frac{85}{81}$ and hence (40) becomes

$$
\begin{aligned}
& \sum_{r=1}^{\left[\frac{k}{\ell}\right]} \frac{(k-1.7 r+2(1.7))^{2}-3(1.7)^{2}}{1.7^{r}(k-1.7 r+4(1.7))_{1.7}^{(2)}(k-1.7 r+1.7)_{1.7}^{\left.\left(\frac{\Gamma-1.7 r+1.7}{1.7}\right]\right)}} \\
& \quad=\frac{85}{81(1.7)^{\left\lceil\frac{k}{1.7}\right\rceil}}-\frac{1}{(k+3(1.7)) k_{1.7}^{\left(\left\lceil\frac{k}{1.7}\right]\right)}}, \quad k=2,3.7,5.4, \ldots
\end{aligned}
$$

Example 3.8 Taking $\ell=3.5$ in (41), we obtain

$$
\begin{aligned}
& \sum_{r=0}^{\infty} \frac{(k+3.5 r)^{3}-3.5^{3}}{3.5^{r}\left((k+3.5 r)^{2}-2(3.5)^{2}\right)_{\ell}^{(2)}(k+3.5 r+3.5)_{3.5}^{\left(\left\lceil\frac{k+3.5 .5 .3 .5}{3.5}\right)\right.}} \\
& \quad=\frac{1}{\left((k-3.5)^{2}-2(3.5)^{2}\right) k_{3.5}^{\left(\left\lceil\Gamma_{3.5}\right]\right)}} .
\end{aligned}
$$


In particular, when $k=9$, above series becomes

$$
\begin{aligned}
& \frac{9^{3}-3.5^{3}}{\left(9^{2}-2(3.5)^{2}\right)_{3.5}^{(2)} 12.5_{3.5}^{(4)}}+\frac{12.5^{3}-3.5^{3}}{3.5\left(12.5^{2}-2(3.5)^{2}\right)_{3.5}^{(2)} 16_{3.5}^{(5)}} \\
& +\frac{16^{3}-3.5^{3}}{3.5^{2}\left(16^{2}-2(3.5)^{2}\right)_{3.5}^{(2)} 19.5_{3.5}^{(6)}}+\cdots=\frac{1}{\left(5.5^{2}-2(3.5)^{2}\right) 9_{3.5}^{(3)}}
\end{aligned}
$$

\section{Concluding remarks}

In the difference equations there are several interesting development, see for example, [4-6], and [8-16]. Recently, in [7], the fractional h-difference equations was studied. In the present work we study the $\ell_{2(\ell)}$ and $c_{0(\ell)}$ solutions of the second order generalized difference equation

$$
\Delta_{\ell}^{2} u(k)+f(k, u(k))=0, \quad k \in[a, \infty), a>0
$$

and we prove the condition for non existence of non-trivial solution.

\section{Competing interests}

The authors declare that they do not have competing interest.

\section{Authors' contributions}

All the authors contributed equally.

\section{Author details}

'Department of Science and Humanities, R.M.D. Engineering College, Kavaraipettai, Tamil Nadu 601 206, India.

${ }^{2}$ Department of Mathematics and Institute for Mathematical Research, University Putra Malaysia, Serdang, Selangor 43400, Malaysia. ${ }^{3}$ Department of Mathematics, Sacred Heart College, Vellore District, Tirupattur, Tamil Nadu 635601, India.

\section{Acknowledgements}

The authors would also like to thank the referee(s) for valuable remarks and suggestions on the previous version of the manuscript.

Received: 5 March 2012 Accepted: 25 June 2012 Published: 12 July 2012

\section{References}

1. Mickens, RE: Difference Equations. Van Nostrand, New York (1990)

2. Kelley, WG, Peterson, AC: Difference Equations. An Introduction with Applications. Academic Press, Boston (1991)

3. Elaydi, SN: An Introduction to Difference Equations, 2nd edn. Springer, New York (1999)

4. Agarwal, RP: Difference Equations and Inequalities. Dekker, New York (2000)

5. Cheng, SS, Patula, WT: An existence theorem for a nonlinear difference equation. Nonlinear Anal. 20, 193-203 (1993)

6. Hartman, P, Wintner, A: On linear difference equations of the second order. Am. J. Math. 72, 124-128 (1950)

7. Ferreira, RAC, Torres, DFM: Fractional $h$-difference equations arising from the calculus of variations. Appl. Anal. Discrete Math. 5, 110-121 (2011)

8. Popenda, J, Schmeidal, E: Some properties of solutions of difference equations. Fasc. Math. 13, 89-98 (1981)

9. Manuel, MMS, Xavier, GBA, Thandapani, E: Theory of generalized difference operator and its applications. Far East J. Math. Sci. 20(2), 163-171 (2006)

10. Manuel, MMS, Xavier, GBA, Thandapani, E: Qualitative properties of solutions of certain class of difference equations. East. J. Math. Sci. 23(3), 295-304 (2006)

11. Manuel, MMS, Xavier, GBA, Chandrasekar, V: Generalized difference operator of the second kind and its application to number theory. Int. J. Pure Appl. Math. 47(1), 127-140 (2008)

12. Manuel, MMS, Xavier, GBA, Dilip, DS, Chandrasekar, V: General partial sums of reciprocals of products of consecutive terms of arithmetic progression. Int. J. Comput. Appl. Math. 4(3), 259-272 (2009)

13. Manuel, MMS, Xavier, GBA: Generalized difference calculus of sequences of real and complex numbers. Int. J. Comput. Numer. Anal. Appl. 6(4), 401-415 (2004)

14. Garner, HG, De Wilde, M, Schmets, J: Analyse Functionnelle, pp. 12-66. Birkhauser, Berlin (1973)

15. Wintner, A: A criterion for nonexistence for $L^{2}$-solutions of nonoscillatory differential equation. J. Lond. Math. Soc. 25 , 347-351 (1950)

16. Wyrwinska, A: On nonexistence of solutions from the class $L_{p}$ for some nonlinear second order differential equation. Fasc. Math. 9, 33-38 (1975) 